\title{
A bulk-driven quasi-floating gate regulated cascode current mirror and its application in squarer circuit
}

\author{
Mounira Bchir, Imen Aloui, and Nejib Hassen \\ Micro-electronics and instrumentation laboratory University of Monastir, Tunisia
}

Corresponding author: Mounira Bchir (e-mail: bchir-mounira@live.fr).

ABSTRACT A regulated cascode current mirror (RGC) and its improved version with bulk driven quasi floating gate technique (BD-QFG) are presented in this paper. The proposed BD-QFG RGC current mirror (CM) is compared with the conventional (GD) RGC CM to show the performance improvement. The conventional and unconventional CM are implemented in Candace Virtuoso using $90 \mathrm{~nm}$ CMOS technology. For input current $\left(\mathrm{I}_{\text {in }}\right)$ varied from 0 to $200 \mu \mathrm{A}$ and for $0.8 \mathrm{~V}$ supply voltage, the simulation results present that the proposed BD-QFG RGC CM has less variation in current transfer error $(0.2 \%)$ as compared to the GD RGC CM (12\%). The output voltage requirement for $200 \mu \mathrm{A}$ input current is respectively $0.7 \mathrm{~V}$ and $0.17 \mathrm{~V}$ for the GD RGC CM and the BD-QFG RGC CM. The power consumption of the proposed circuit is $22.71 \mu \mathrm{W}$ which is $0.15 \mu \mathrm{W}$ higher than the GD RGC $(22.56 \mu \mathrm{W})$. The total harmonic distortion (THD) of the proposed circuit is $0.4 \%$ which is $1.1 \%$ less than the conventional circuit (1.5\%). All these improvements in the proposed BD-QFG RGC CM are attained at a cost of 0.05 $\mathrm{GHz}$ reduction in frequency $(2.31 \mathrm{GHz})$. The minimum supply voltage of BD-QFG RGC CM and GD RGC CM is $0.4 \mathrm{~V}$ and $0.8 \mathrm{~V}$ respectively. The designed chip of the proposed BD-QFG RGC CM occupies an area of $29.4 \mu \mathrm{m}^{2}$. To show the workability of the proposed circuit, a new squarer circuit has been presented.

INDEX TERMS BD-QFG - GD - Current mirror - Squarer

\section{INTRODUCTION}

Low voltage and low power circuit is demanded in portable electronics to extend the battery lifetime. For analog circuits, higher threshold voltage CMOS technology imposes a serious degrading circuit performance [1].

In the literature, diverse techniques unconventional have been developed to construct the low supply voltage and the low consumption CMOS circuits. The important techniques are the floating-gate (FG) [2], the quasi- floating-gate (QFG) [3] and the bulk-driven (BD) [4]. These techniques have many advantages and disadvantages. The disadvantages of the unconventional techniques (FG, QFG) in comparison to the conventional technique (GD) are the reduction of the effective transconductance, the high occupied chip area and the lower output impedance. Although, these techniques (FG, QFG) offer the possibility of multiple inputs. Moreover, the threshold voltage can be reduced in the FG and QFG techniques [5]. For the BD technique, the threshold voltage is removed, and it can process DC signals. The disadvantage of the BD MOST is the lower transconductance than the FG, QFG and GD MOST. A two new MOS techniques BulkDriven Floating-Gate (BD-FG) and Bulk-Driven QuasiFloating-Gate (BD-QFG) are firstly presented in 2014 [6], experimentally verified and published in the literature [7]. The BD-QFG technique has revealed all the advantages of $\mathrm{BD}$ and $\mathrm{QFG}$ techniques and suppress their limitations. The symbol of the BD-QFG MOST is shown in Fig. 1.a, and their equivalent circuit is exposed in Fig. 1.b[14]. In this technique, the input signal $\left(\mathrm{V}_{\text {in }}\right)$ is applied to the quasi-floating gate and to the bulk terminal through the capacitance $\mathrm{C}_{\mathrm{in}}$. The quasifloating-gate terminal of the BD-QFG MOST is connected by a very high value resistor $\left(\mathrm{R}_{\mathrm{b}}\right)$ to an appropriate bias voltage $\left(\mathrm{V}_{\text {bias }}\right)$. Practically, this resistance $\left(\mathrm{R}_{\mathrm{b}}\right)$ is implemented via transistor $\mathrm{M}_{\mathrm{R}}$ operating in the cutoff region. The current mirror (CM) structure is an important circuit in many applications, such as the conveyors, the multiplier, the comparator [1], the OTA, the ADC [8], [9] and the DAC. Various researches have been reported to attain the perfect performance of the $\mathrm{CM}$ circuit. The characteristic of the $\mathrm{CM}$ has a direct impact on the efficiency of the integrated circuits [10]-[12]. The CM presented in [13] is characterized by a low input impedance and a high output impedance. These types of $\mathrm{CM}$ are needed to minimize the loading effect. But it has a very high supply voltage and power operation. In [14], using the unconventional technique (BD), a CM circuit is introduced to surmount the limitation of the high supply voltage of the technique GD. This circuit presented an amelioration in terms of power consumption and supply voltage, but it has low bandwidth. High bandwidth in a CM is an important characteristic of increasing the speed of devices. In [15], the CM employs the BD-QFG technique. It presents amelioration in terms of low supply voltage and low input impedance, but it suffers from lowered output impedance and a high number of transistors. Based on the advantages of the unconventional technique (BD-QFG), a new regulated cascode $\mathrm{CM}$ circuit is proposed. The interesting performances of the proposed circuit are low voltage supply, high bandwidth, low consumption, and high output impedance. The proposed circuit is employed to realize a high-performance squarer circuit. This paper is arranged as follows. Section 2 presents the proposed BDQFG RGC CM and calculates its significant performances. 
Simulation results are presented in Section 3. Section 4 presents the application of the proposed CM. Finally, Section 5 concludes the paper.

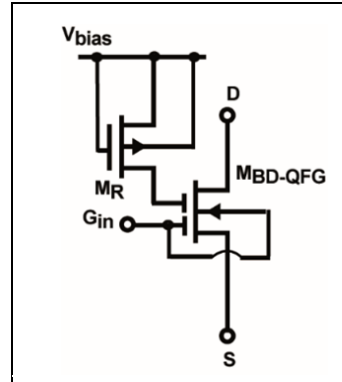

(a)

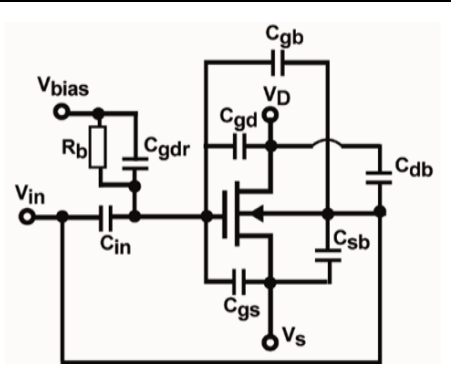

(b)
Fig. 1. T ransistor NMOS BD-QFG: (a) symbol, (b) equivalent circuit

\section{Proposed current mirror}

\subsection{Circuit description}

In this part, the operation and implementation of the conventional and unconventional CM are discussed. The conventional RGC CM proposed in [16] is shown in Fig. 2.a. This circuit is composed of a simple $\mathrm{CM}\left(\mathrm{M}_{1}, \mathrm{M}_{2}\right)$ and an amplification stage.

The amplification stage is consisting of a transistor $\mathrm{M}_{4}$, a current source $\mathrm{I}_{\mathrm{B} 1}$, and a cascode transistor $\mathrm{M}_{3}$. This structure provides a high output impedance owing to the negative feedback offered by the transistor $\mathrm{M}_{4}$ and the bias current $\mathrm{I}_{\mathrm{B}}$. This circuit performs a low supply voltage and high output impedance.

Due to the augmented demand for smaller electronic devices, reducing the supply voltage, removing the threshold voltage, reducing the input impedance and lowering the power consumption becomes necessary. Fig.2.b show the proposed RGC CM. It consists of modified the two transistors $\left(\mathrm{M}_{1}, \mathrm{M}_{2}\right)$ based on the GD technique by two transistors $\mathrm{M}_{1}$ and $\mathrm{M}_{2}$ based on the BD-QFG technique and to replace the bias source $\mathrm{I}_{\mathrm{B} 1}$ with an active current source $\mathrm{M}_{5}$.

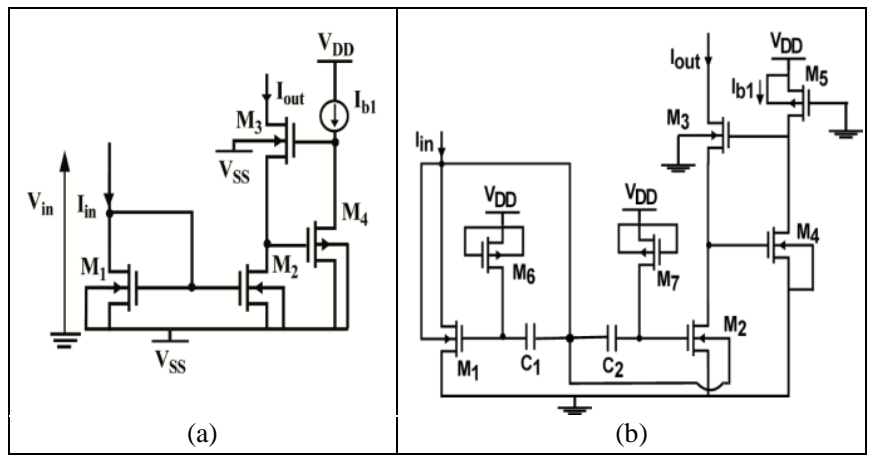

Fig. 2. RGC CM: (a): the GD circuit, (b): the proposed BD-QFG circuit

The unconventional BD-QFG transistor has a high transconductance near to the transconductance of the GD technique, and it can be presented [6], [7]:

$$
\begin{aligned}
& g_{m 1 B D-Q F G}=g_{m 1 \text { eff }}+g_{m b 1} \\
& g_{m 2 B D-Q F G}=g_{m 2 e f f}+g_{m b 2}
\end{aligned}
$$

\subsection{Small signal analysis}

\subsubsection{Output impedance}

The small signal analysis for calculating the output impedance of the proposed circuit, is presented in Fig. 3.

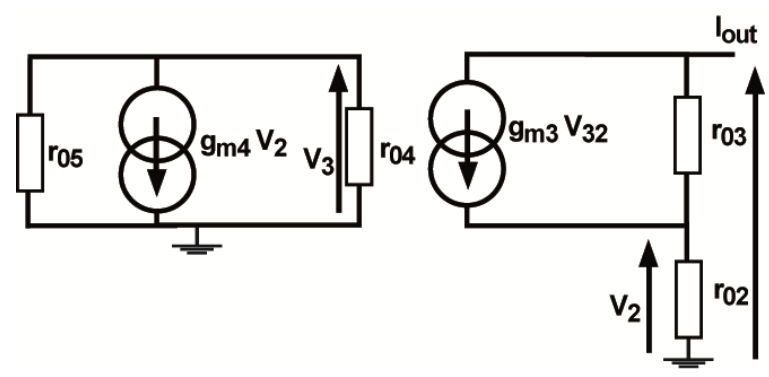

Fig. 3. Small signal analysis for calculating the output impedance

At node2:

$$
\begin{gathered}
V_{2}=i_{\text {out }} r_{02} \\
V_{2}=\frac{V_{\text {out }} r_{02}+r_{02} r_{03} g_{m 3} V_{32}}{r_{02}+r_{03}}
\end{gathered}
$$

At node 3:

$$
V_{3}=-g_{m 4} \frac{r_{04} r_{05}}{r_{04}+r_{05}} V_{2}
$$

Based on these three expressions, the equation for the output resistance is given by:

$$
\begin{gathered}
r_{\text {out }}=\frac{V_{\text {out }}}{i_{\text {out }}}=r_{02}+r_{03}+r_{02} g_{m 3} r_{03}\left(1+\frac{g_{m 4} r_{04} r_{05}}{r_{04}+r_{05}}\right) \\
r_{\text {out }} \approx r_{02}\left(g_{m 3} r_{03}\right) \frac{g_{m 4} r_{04} r_{05}}{r_{04}+r_{05}}
\end{gathered}
$$

From Eq. (1) and (8), it can be noticed that the output impedance of the BD-QFG RGC circuit is near to the conventional GD RGC circuit.

\subsubsection{Bandwidth:}

For calculating the bandwidth, the small signal analysis is presented in Fig.4.

Assume: $C_{x}=C_{1}+C_{g s 1}, \quad C_{y}=C_{b s 1}+C_{g d 1}, C_{z}=C_{2}+C_{g s 2}+C_{g d 2}$, $C_{A}=C_{x}+C_{y}+C_{z} \quad, \quad g_{m B D-Q F G 1}=g_{m e f f 1}+g_{m b 1} \quad$ and $g_{m B D-Q F G f 2}=g_{\text {meff } 2}+g_{m b 2}$.

At node1: 


$$
i_{i n}=\left(g_{m B D-Q F G 1}+s C_{A}\right) V_{1}
$$

At node2:

$$
V_{2}=\frac{\left(g_{m 3}+s C_{g s 3}\right) V_{3}-g_{m B D-Q F G 2} V_{1}}{s\left(C_{g s 3}+C_{g s 4}\right)}
$$

$$
i_{\text {out }}=g_{m 3} V_{3}
$$

From Eq. (10) and (11), the expression of $\mathrm{V}_{2}$ is found as:

$$
V_{2}=\frac{\left(g_{m 3}+s C_{g s 3}\right)\left(\frac{i_{\text {out }}}{g_{m 3}}\right)-g_{m B D-Q F G 2} V_{1}}{s\left(C_{g s 3}+C_{g s 4}\right)}
$$

The current transfer function is expressed by:

At node3:

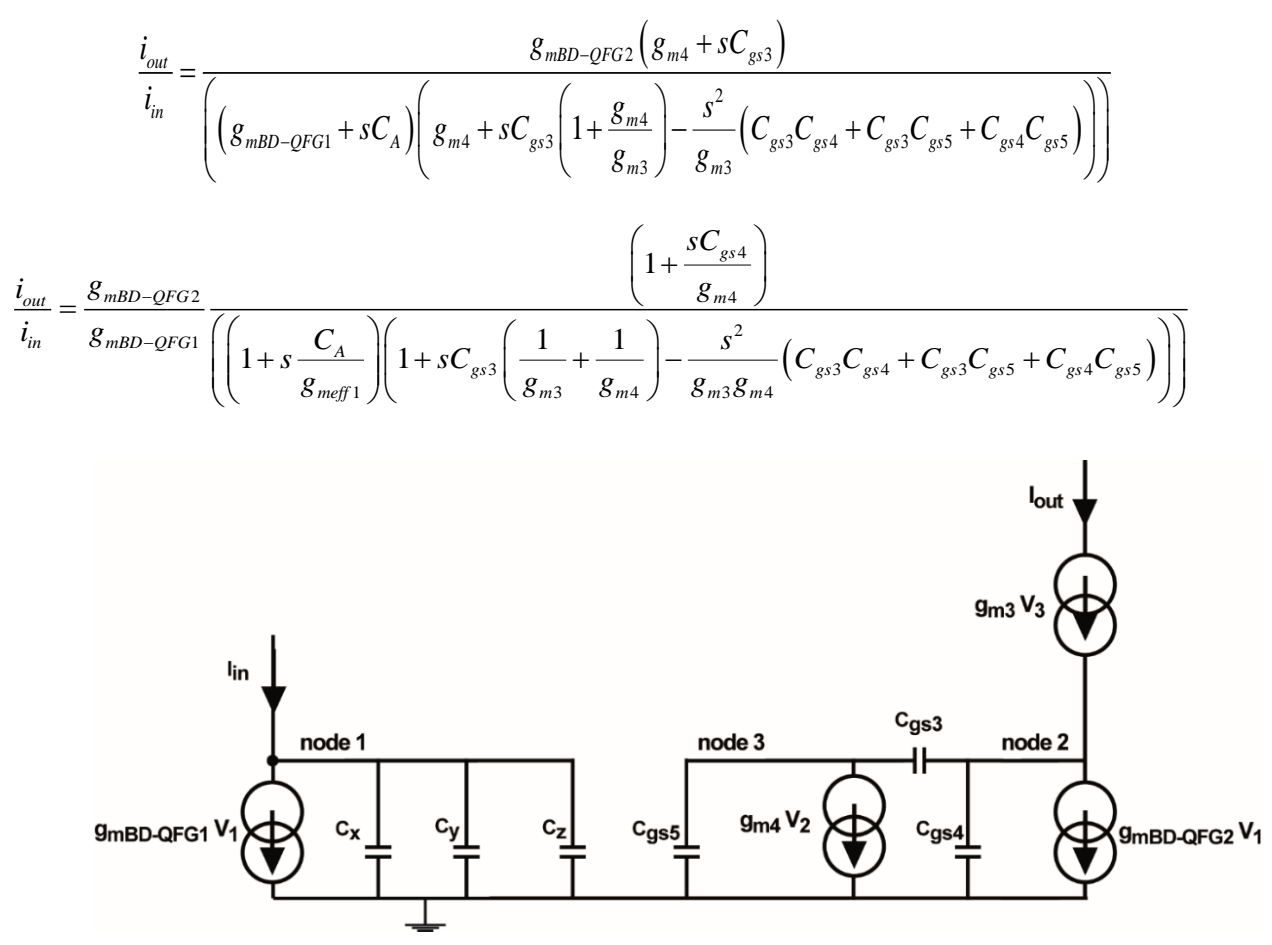

Fig. 4. Small signal analysis for calculating the bandwidth

Assume: $C_{A}=C_{1}+C_{g s 1}+C_{b s 1}+C_{g d 1}+C_{2}+C_{g s 2}$

The equation for the transfer function is given as follows:

$$
\frac{i_{\text {out }}}{i_{\text {in }}}=\frac{g_{m B D-Q F G 2}}{g_{m B D-Q F G 1}} \frac{\left(1+j \frac{\omega}{\omega_{01}}\right)}{\left(1+j \frac{\omega}{\omega_{02}}\right)\left(1+\frac{2 \varepsilon s}{\omega_{03}}+\frac{\omega^{2}}{\omega_{03}^{2}}\right)}
$$

The expressions for the gain and the pulse are expressed as follows:

$$
\begin{array}{r}
A_{0}=\frac{g_{m B D-Q F G 2}}{g_{m B D-Q F G 1}} \\
\omega_{01}=\frac{g_{m 4}}{C_{g s 4}} \\
\omega_{02}=\frac{g_{m B D-Q F G 1}}{C_{A}}
\end{array}
$$


conventional and unconventional RGC CM shown in Fig. 2 have been simulated in the same environment to provide an appropriate comparison. The dimensions of transistors and element values of the GD RGC and the BD-QFG RGC CM are identical. For the conventional RGC CM, the current source $\mathrm{I}_{\mathrm{B} 1}$ has been designed by using a transistor $\mathrm{M}_{5}$.

Fig. 5 shows the current transfer characteristic of the conventional GD RGC CM and the proposed BD-QFG RGC for different values of $\mathrm{V}_{\mathrm{DD}}(0.4 \mathrm{~V}, 0.6 \mathrm{~V}$, and $0.8 \mathrm{~V})$ and Iin ranging from 0 to $200 \mu \mathrm{A}$. As it is seen, the proposed circuit exhibits excellent current tracking between input and output currents. The maximum current transfer error for the conventional and unconventional $\mathrm{CM}$ circuits for $0.8 \mathrm{~V}$ is respectively $12 \%$ and $0.2 \%$.

It can be remarked that the BD-QFG RGC CM shows an acceptable current pursuit between the input and the output. In Fig.6, the output current of the GD RGC and of the BDQFG RGC is plotted for different input current values from $0 \mu \mathrm{A}$ to $200 \mu \mathrm{A}$ in steps of $50 \mu \mathrm{A}$ and for the output voltage ranging from 0 to $0.8 \mathrm{~V}$. The output voltage requirement for the conventional and the unconventional $\mathrm{CM}$ circuits is respectively $0.7 \mathrm{~V}$ and $0.17 \mathrm{~V}$.

The frequency responses of the RGC CM based on different techniques are compared in Fig. 7. The maximum bandwidth for the GD RGC and the BD-QFG RGC CM is respectively $2.36 \mathrm{GHz}$ and $2.31 \mathrm{GHz}$, and the power consumption is respectively $22.56 \mu \mathrm{W}$ and $22.71 \mu \mathrm{W}$. The bandwidth value of the proposed circuit is very near to the bandwidth value of the GD-MOST.

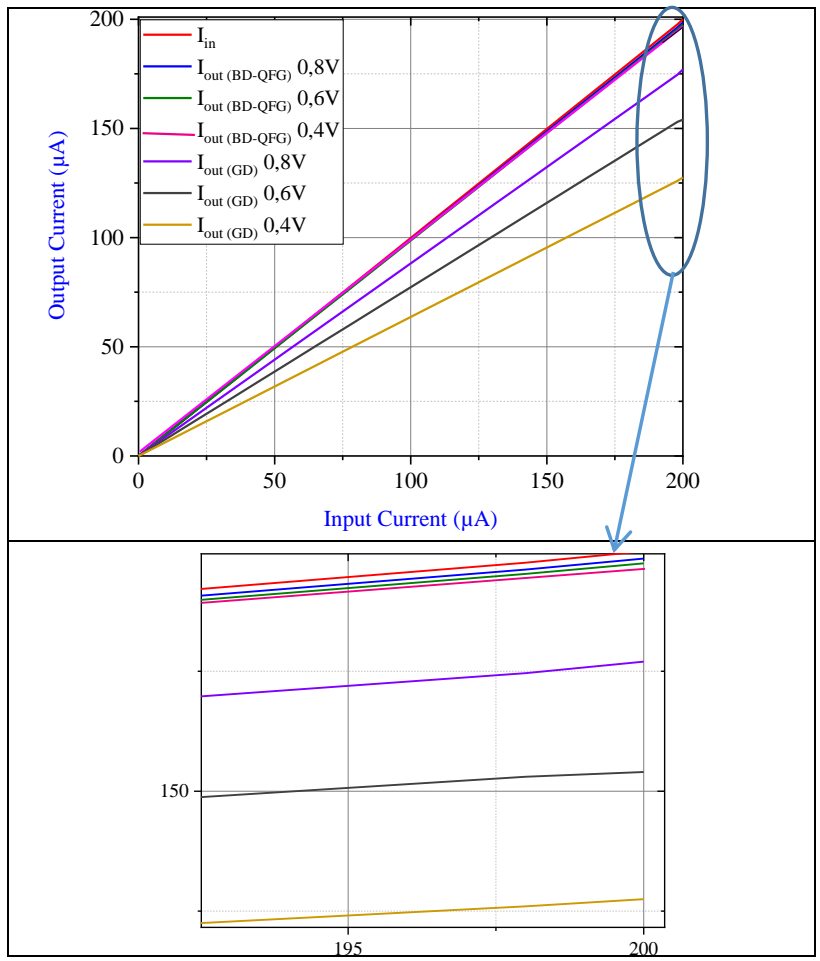

Fig.5. Current transfer characteristics of the GD RGC and the BD-QFG RGC CM for different supply voltage

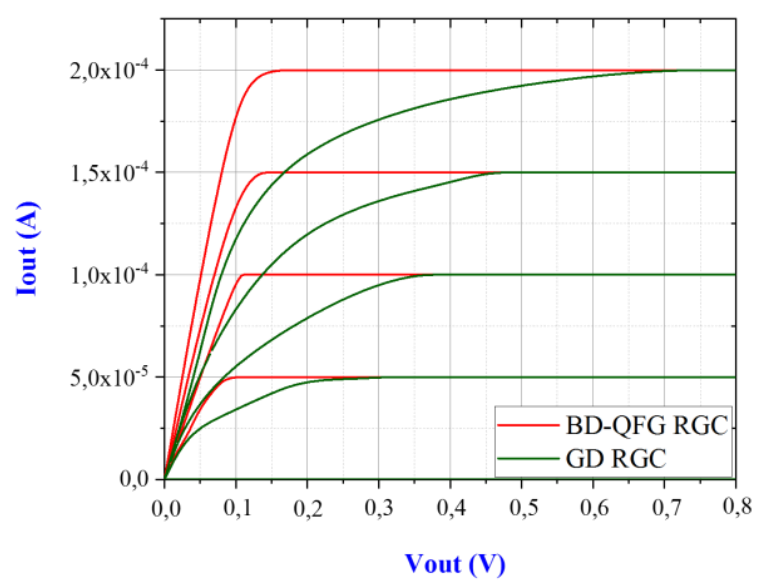

Fig.6. output voltage characteristic of GD RGC and BD-QFG RGC CM

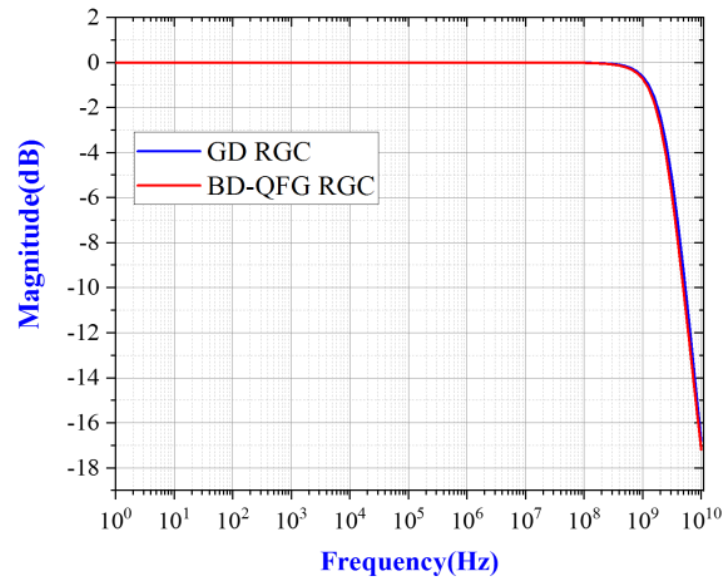

Fig. 7. Frequency response of the conventional and unconventional RGC $\mathrm{CM}$

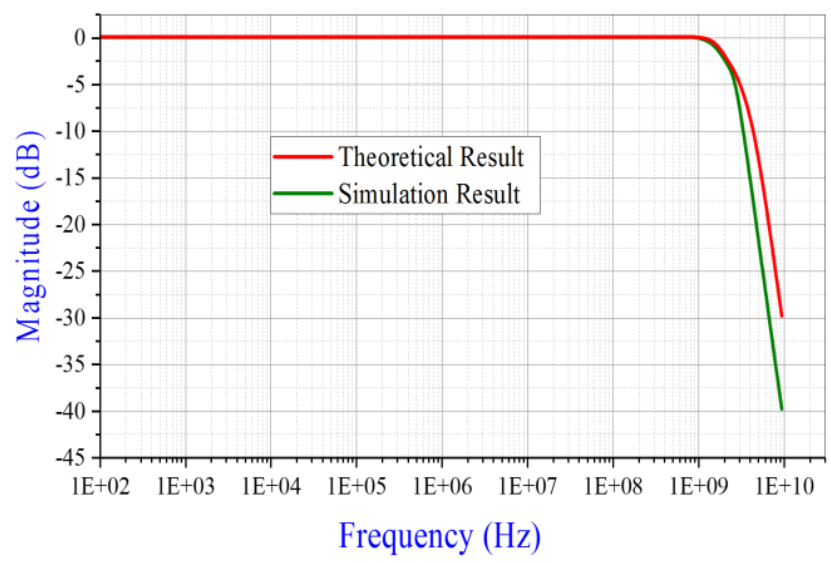

Fig. 8. The simulated and the calculated results of the frequency response of the proposed CM

To demonstrate the maximum frequency, the transfer function shown in Eq. (20) is calculated by MATLAB and presented in the Fig. 8. It is clear that the simulated and the calculated results are in good agreement. Fig. 9 shows the output impedance, where their value is very near. The output impedance values achieved by the conventional and the unconventional $\mathrm{CM}$ are respectively $14.75 \mathrm{G} \Omega$ and $15 \mathrm{G} \Omega$. 
Fig. 10 present the resulted output current of the conventional and unconventional RGC CM for a sinusoidal input current signal with a frequency of $10 \mathrm{KHz}$ and a peakto-peak value of $100 \mu \mathrm{A}$, the total harmonic distortion (THD) is respectively $1.5 \%$ and $0.4 \%$. The Monte-Carlo analysis is presented of 500 runs for an input current $200 \mu \mathrm{A}$ for some important specifications including the bandwidth, the current transfer error, and the output impedance. From Fig. 11, it is seen that the proposed CM does not present an important degradation within the mentioned specifications.

The layout of the proposed BD-QFG RGC CM has been done by using $90 \mathrm{~nm}$ process technology with cadence virtuoso. The total chip area was $4.2 \mu \mathrm{m} \times 7 \mu \mathrm{m}\left(29.4 \mu \mathrm{m}^{2}\right)$. The layout simulation is shown in Fig. 12. The BD-QFG technique shows the best performance in the current mirror design in terms of low supply voltage, low current transfer error, low output voltage and low THD value. While the other parameters (frequency, output impedance, and consumption) are close for both techniques. The comparative study between the BD-QFG RGC CM and GD RGC CM is summarized in Table 1.

The validity of the layout is confirmed by using the postlayout simulation. Table. 2 present a comparison between the post-layout and the pre-layout simulations. From this table, it is clear that the variations between the pre-layout and the post-layout simulations are very weak. The simulation results of the proposed BD-QFG RGC CM are presented with other works in Table. 3.

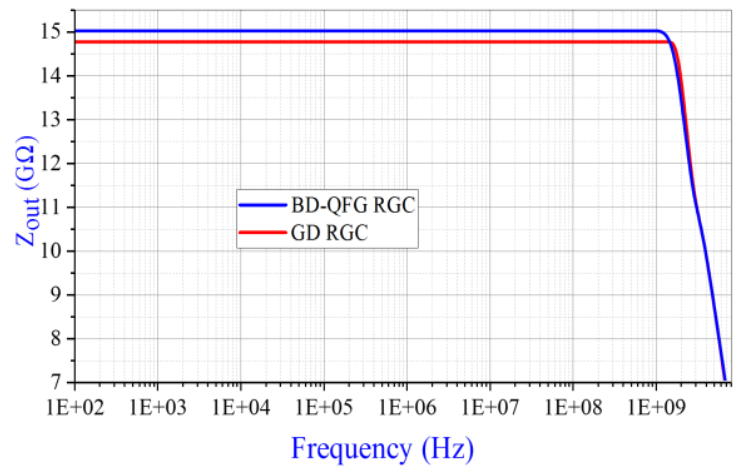

Fig. 9. Output impedance of the conventional and unconventional RGC $\mathrm{CM}$

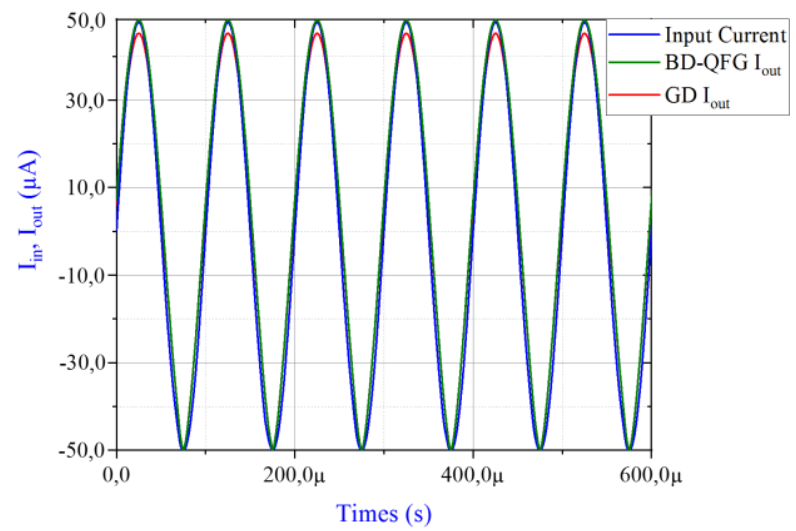

Fig. 10. The transient response for a sinusoidal input of the conventional and unconventional RGC CM

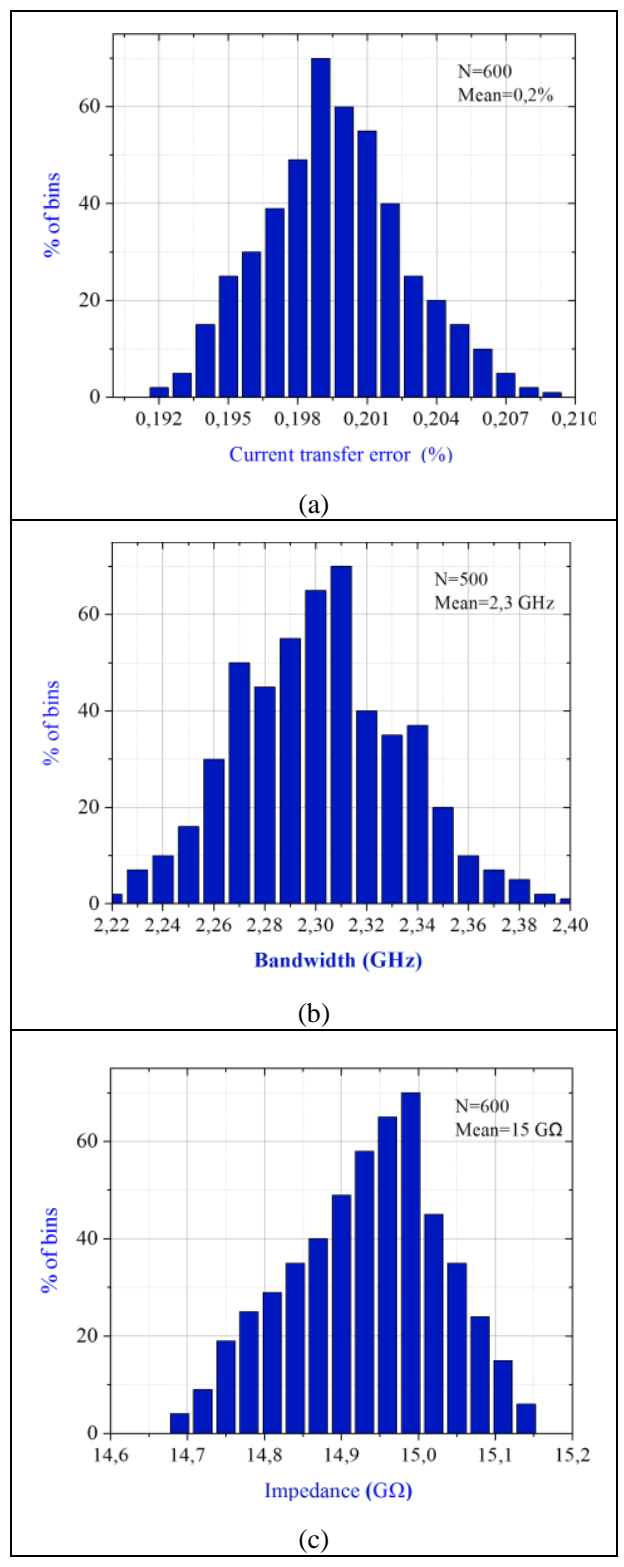

Fig. 11. Monte-Carlo analysis for the proposed BD-QFG RGC current mirror, (a): Current transfer error, (b): Bandwidth, (c): Output impedance

Table. 1. Comparative analysis of proposed BD-QFG RGC CM and GD RGC CM

\begin{tabular}{|c|c|c|}
\hline & GD RGC & BD-QFG RGC \\
\hline Supply voltage (V) & 0.8 & 0.8 \\
\hline Output voltage $(\mathrm{V})$ & $0.7($ for $200 \mu \mathrm{A})$ & 0.17 (for $200 \mu \mathrm{A})$ \\
\hline $\begin{array}{c}\text { Power Consumption } \\
(\mu \mathrm{W})\end{array}$ & 22.56 & 22.71 \\
\hline Bandwidth $(\mathrm{GHz})$ & 2.36 & 2.31 \\
\hline Current range $(\mu \mathrm{A})$ & 200 & 200 \\
\hline $\begin{array}{c}\text { Current transfer error } \\
(\%)\end{array}$ & $12 \%$ & $0.2 \%$ \\
\hline Output impedance $(\mathrm{G} \Omega)$ & 14.75 & 15 \\
\hline $\begin{array}{c}\text { Minimum supply voltage } \\
(\mathrm{V})\end{array}$ & 0.8 & 0.4 \\
\hline THD & $1.5 \%$ & $0.4 \%$ \\
\hline
\end{tabular}


Table. 2. Difference between the pre-layout and the post-layout simulations of the proposed BD-QFG RGC CM

\begin{tabular}{|c|c|c||c|}
\hline Parameter & $\begin{array}{c}\text { Pre- } \\
\text { layout }\end{array}$ & $\begin{array}{c}\text { Post- } \\
\text { Layout }\end{array}$ & $\begin{array}{c}\text { Percentage } \\
\text { variation }\end{array}$ \\
\hline Bandwidth $(\mathrm{G} \mathrm{Hz})$ & 2.31 & 2.29 & $0.86 \%$ \\
\hline THD & $0.4 \%$ & $0.4 \%$ & $0 \%$ \\
\hline Output impedance $(\mathrm{G} \Omega)$ & 15 & 14.92 & $0.53 \%$ \\
\hline Power Consumption $(\mu \mathrm{W})$ & 22.79 & 22.79 & $0 \%$ \\
\hline
\end{tabular}

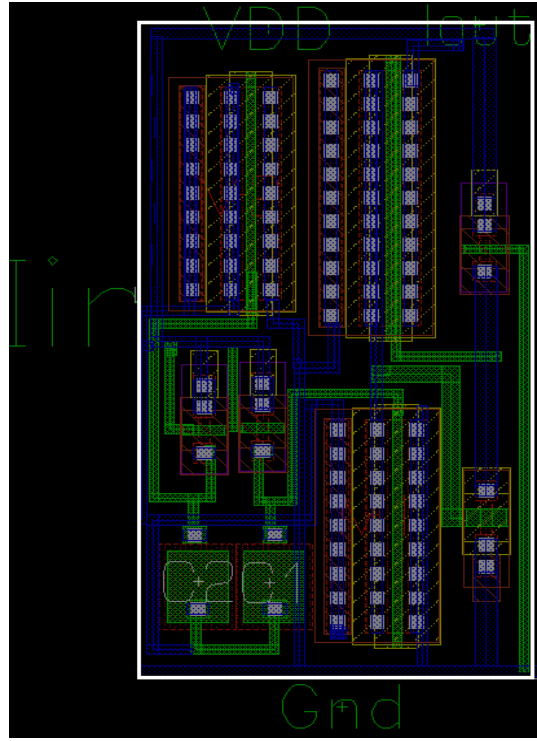

Fig. 12. Layout of the proposed BD-QFG RGC CM

Table. 3. Comparative analysis of proposed BD-QFG RGC CM

\begin{tabular}{|c|c|c|c|c|c|}
\hline & {$[17]$} & {$[18]$} & {$[19]$} & {$[20]$} & $\begin{array}{c}\text { BD-QFG } \\
\text { CM }\end{array}$ \\
\hline $\begin{array}{c}\text { Technology } \\
(\mathrm{m})\end{array}$ & $0.13 \mu$ & $\begin{array}{c}0.18 \\
\mu\end{array}$ & $\begin{array}{c}0.18 \\
\mu\end{array}$ & $\begin{array}{c}0.18 \\
\mu\end{array}$ & $90 \mathrm{n}$ \\
\hline $\begin{array}{c}\text { Supply } \\
\text { Voltage }(\mathrm{V})\end{array}$ & \pm 0.5 & \pm 0.2 & 1 & 0.9 & 0.8 \\
\hline $\begin{array}{c}\text { Power } \\
\text { Consumptio } \\
\mathrm{n}(\mathrm{W})\end{array}$ & $0.83 \mathrm{~m}$ & $\begin{array}{c}0.34 \\
9 \mathrm{~m}\end{array}$ & - & $\begin{array}{c}154 \\
\mu\end{array}$ & $22.71 \mu$ \\
\hline $\begin{array}{c}\text { Bandwidth } \\
(\mathrm{Hz})\end{array}$ & $1.52 \mathrm{G}$ & $\begin{array}{c}285 \\
\mathrm{M}\end{array}$ & $\begin{array}{c}402 \\
\mathrm{M}\end{array}$ & $\begin{array}{c}181 \\
\mathrm{M}\end{array}$ & $2.31 \mathrm{G}$ \\
\hline $\begin{array}{c}\text { Current } \\
\text { range }(\mu \mathrm{A})\end{array}$ & 500 & 250 & $\begin{array}{c}100 \\
0\end{array}$ & 100 & 200 \\
\hline $\begin{array}{c}\text { Current } \\
\text { transfer } \\
\text { error }(\%)\end{array}$ & 3.6 & - & 0.28 & 0.6 & 0.2 \\
\hline $\begin{array}{c}\text { Output } \\
\text { impedance } \\
(\Omega)\end{array}$ & $117 \mathrm{~K}$ & 19.5 & 10.5 & $1 \mathrm{M}$ & $15 \mathrm{G}$ \\
\hline Area $\left(\mathrm{m}^{2}\right)$ & - & - & - & - & $29.4 \mu$ \\
\hline
\end{tabular}

\section{Application of the $\mathrm{CM}$}

\subsection{BD-QFG squarer circuit}

The squarer circuit is an important circuit in the design of many integrated circuits. It is can be used in many applications such as the current multipliers, the automatic gain controlling, the frequency doubles and the modulator circuit.

The implementation of the conventional and unconventional squarer circuit is discussed in this part. The conventional squarer circuit presented in [21] is shown in Fig. 13.a. This structure is composed of simple current mirrors based on the conventional (GD) technique. According to the abovementioned advantage of the proposed BD-QFG RGC CM, a new squarer circuit is proposed. Fig. 13.b shows the proposed BD-QFG squarer circuit.

The transconductance of the BD-QFG MOST operating in the saturation region is determined by [6]:

$$
\begin{aligned}
& g_{m B D-Q F G}=g_{m b}+g_{\text {meff }} \\
& g_{m, e f f}=\frac{C_{\text {in }}}{C_{\text {total }}} g_{m} \\
& g_{m b}=\frac{C_{B C}}{C_{G C}} g_{m}
\end{aligned}
$$

Where $\mathrm{C}_{\text {Total }}$ is the capacitance seen from the $\mathrm{QFG}, \mathrm{C}_{\mathrm{in}}$ is the input capacitance between the QFG and the input-terminal, $\mathrm{C}_{\mathrm{GC}}$ is the gate channel capacitance, and $\mathrm{C}_{\mathrm{BS}}$ is the bulk channel capacitance. Using Eq. (21) to Eqs. (23) the transconductance of the BD-QFG MOST can be written as:

$$
g_{m B D-Q F G}=C_{L} g_{m}
$$

Assume that $C_{L}=\frac{C_{B C}}{C_{G C}}+\frac{C_{i n}}{C_{\text {tota }}}$, the equation of the transconductance can be expressed as:

$$
g_{m B D-Q F G}=\sqrt{2 \beta I_{D}}
$$

Where $\beta=\mu_{0} C_{o x}(W / L)$ present the transconductance parameter, $\mathrm{W} / \mathrm{L}$ present the transistor dimension, $\mathrm{C}_{\mathrm{ox}}$ present the oxide gate capacitance and $\mu_{0}$ present the electron mobility.

Using the Eq. (25) and the Eq. (24), the drain current of BDQFG MOS is given by:

$$
I_{D}=C_{L}^{2} \frac{\beta}{2}\left(V_{G S}-V_{T H}\right)^{2}
$$

The threshold voltage $\left(\mathrm{V}_{\mathrm{TH}}\right)$ is removed in BD-QFG technique [6]. The equation of $\mathrm{V}_{\mathrm{GS}}$ can be presented as:

$$
V_{G S}=\sqrt{\frac{2 I_{D}}{\beta C_{L}^{2}}}
$$

The unconventional transistors $\mathbf{M}_{8}, \mathrm{M}_{15}, \mathrm{M}_{9}, \mathrm{M}_{1}$ based in $\mathrm{BD}-\mathrm{QFG}$ technique and in saturation region form a translinear loop. By applying the MTL principle, Eq. (28) can be written as: 


$$
V_{G S 8}+V_{G S 15}=V_{G S 9}+V_{G S 1}
$$

Using Eq. (26), Eq. (27) can be written as

$$
\sqrt{\frac{2 I_{D 8}}{\beta_{8} C_{L 8}^{2}}}+\sqrt{\frac{2 I_{D 15}}{\beta_{15} C_{L 15}^{2}}}=\sqrt{\frac{2 I_{D 9}}{\beta_{9} C_{L 9}^{2}}}+\sqrt{\frac{2 I_{D 1}}{\beta_{1} C_{L 1}^{2}}}
$$

From the circuit schematic show in Fig. 13.b. We have

$$
\begin{aligned}
& I_{B 1}=I_{D 8}=I_{D 15} \\
& I_{D 9}=I_{D 1}=I_{D 4}+I_{i n}
\end{aligned}
$$

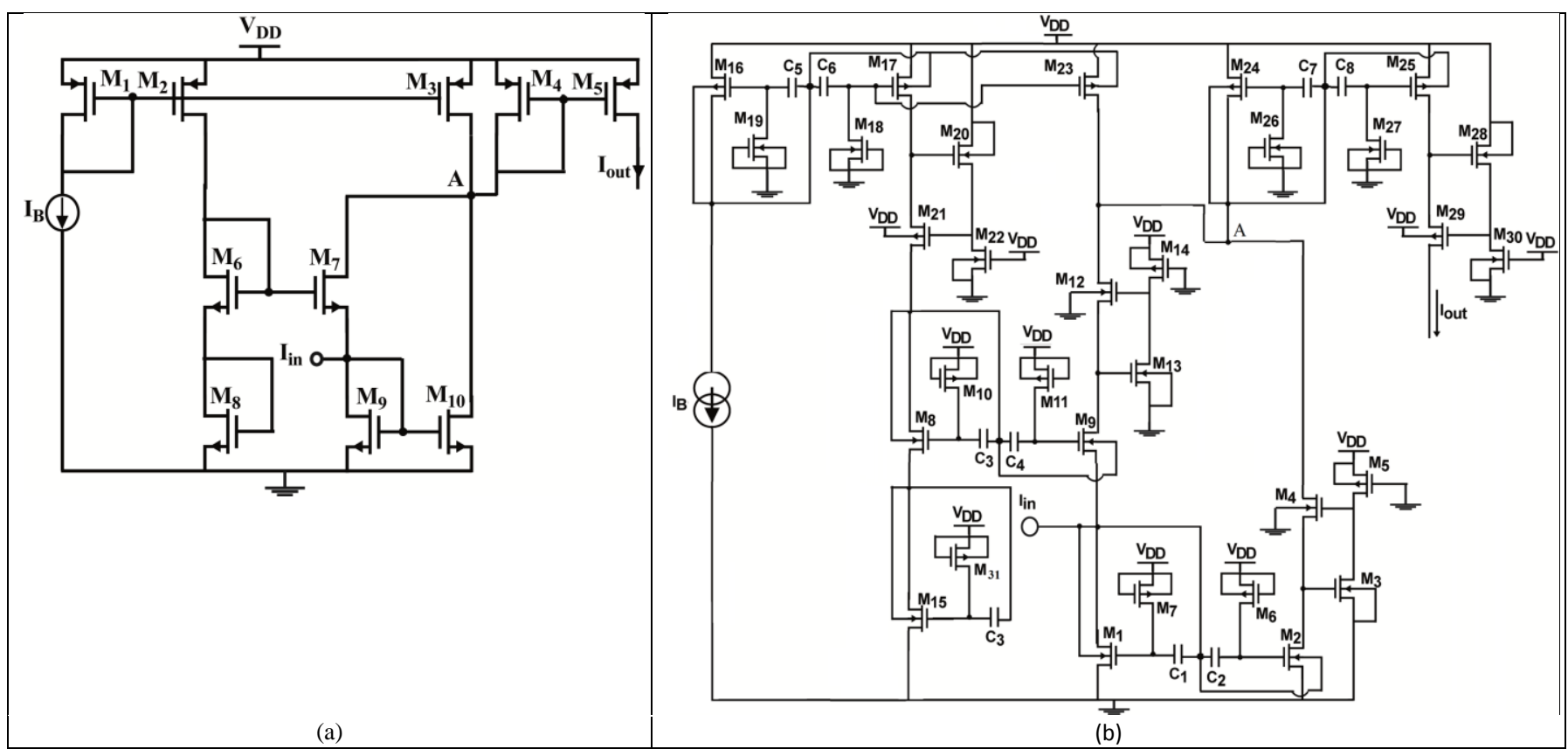

Fig. 13. Squarer circuit, (a): Conventional, (b): Proposed

Assuming the dimension of the transistors and the valor of the capacity $\mathrm{C}_{\mathrm{L}}$ satisfy the condition

$$
\beta_{8} C_{L 8}^{2}=\beta_{15} C_{L 15}^{2}=2 \beta_{9} C_{L 9}^{2}=2 \beta_{1} C_{L 1}^{2}=2 \beta C_{L}^{2}
$$

Using Eqs. (30) to (32), the Eq. (29) can be expressed by

$$
2 \sqrt{\frac{I_{B}}{\beta C_{L}^{2}}}=\sqrt{\frac{2 I_{D 9}}{\beta C_{L}^{2}}}+\sqrt{\frac{2\left(I_{D 4}+I_{\text {in }}\right)}{\beta C_{L}^{2}}}
$$

Squaring Eq. (33) two times we get

$$
32 I_{B} I_{D 8}=16 I_{B}^{2}+4 I_{\text {in }}^{2}-16 I_{B} I_{\text {in }}
$$

Writing the Kirchhoff current law at node A results

$$
I_{\text {out }}+I_{B}=I_{D 2}+I_{D 9}
$$

Using Eq. (35) and Eq. (36), the output current of the proposed squarer circuit is expressed by

$$
I_{\text {out }}=\frac{I_{\text {in }}^{2}}{4 I_{B}}
$$

The simulation results of the conventional and the proposed squarer circuit are simulated through Cadence simulator using $90 \mathrm{~nm}$ CMOS technology parameters with $0.8 \mathrm{~V}$ supply voltage. The conventional and the proposed square circuit have been verified in the same environment to provide a proper comparison.
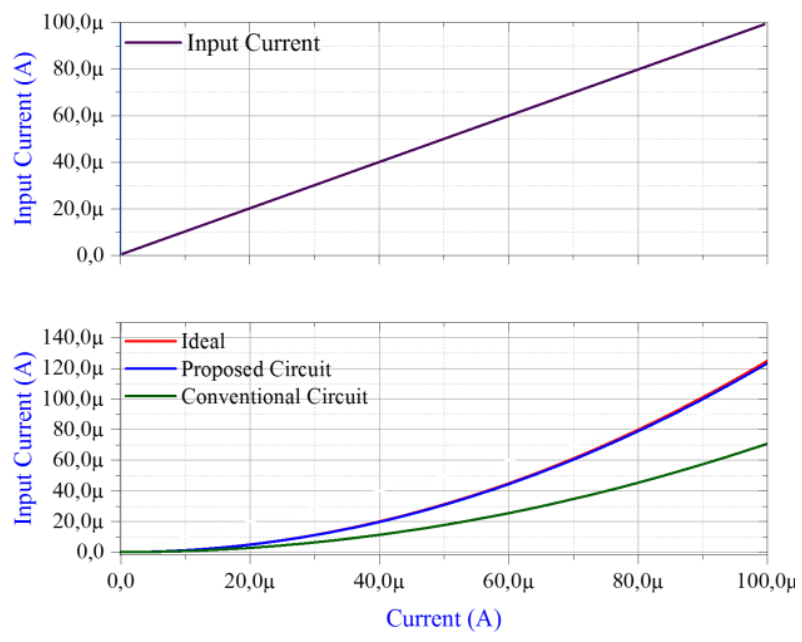

Fig. 14. The DC analysis of the squarer circuit

\subsection{Simulation results}


The bias current $\left(\mathrm{I}_{\mathrm{B}}\right)$ is $20 \mu \mathrm{A}$, the input current $\left(\mathrm{I}_{\text {in }}\right)$ is varied from $0 \mu \mathrm{A}$ to $100 \mu \mathrm{A}$. The DC analysis of the conventional and the proposed squarer circuit is presented in Fig. 14 and the transient analysis is shown in Fig. 15 with an input signal triangular for $1 \mathrm{MHz}$ frequency and for $100 \mu \mathrm{A}$ amplitude. The Nonlinearity Errors analysis of the conventional and the proposed squarer circuit are respectively $41 \%$ and $0.5 \%$. The simulations of frequency show in Fig. 16 a -3 dB are 320 $\mathrm{MHz}$ and $1.2 \mathrm{GHz}$ respectively of the GD and the proposed BD-QFG squarer circuit.

Monte Carlo analysis of the proposed squarer circuit of the bandwidth has been presented in Fig. 17. The simulation results of the proposed BD-QFG squarer circuit are presented with other works in Table. 4.
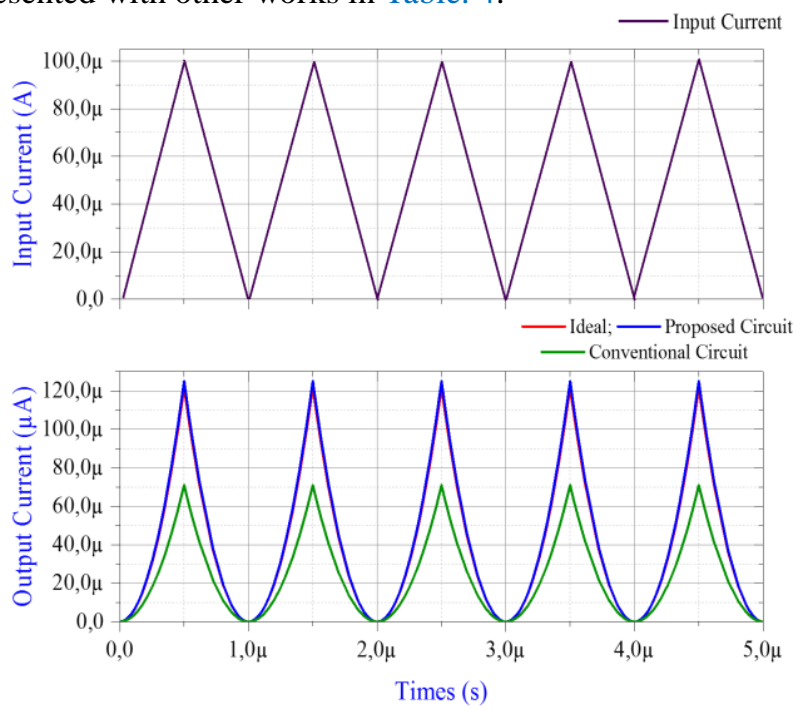

Fig. 15. The transient response of the squarer circuit

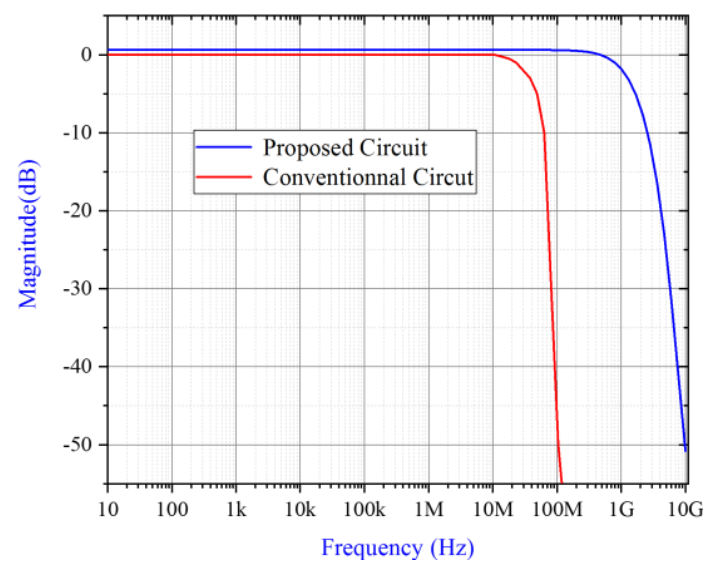

Fig. 16. Frequency characteristics of the squarer circuit

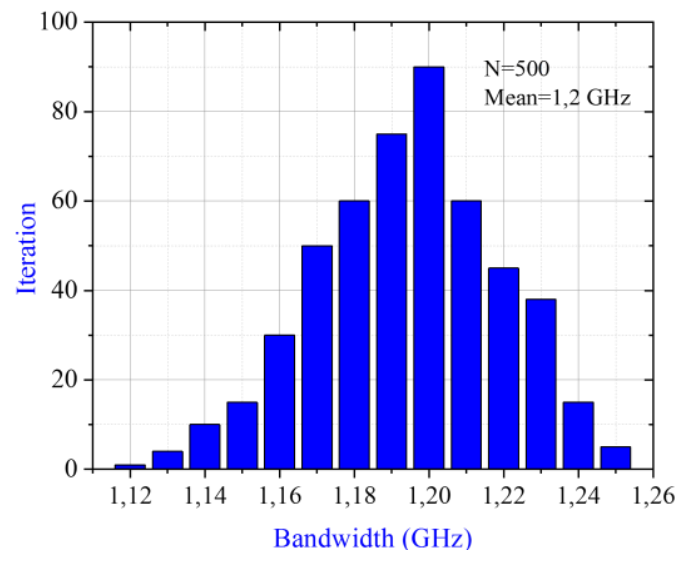

Fig. 17. Monte-Carlo analysis for the proposed squarer circuit

Table. 4. Comparative analysis of proposed squarer circuit

\begin{tabular}{|c|c|c|c|c|c|}
\hline & {$[22]$} & {$[21]$} & {$[23]$} & $\begin{array}{c}\text { Conve } \\
\text { ntional } \\
\text { circuit }\end{array}$ & $\begin{array}{c}\text { Proposed } \\
\text { circuit }\end{array}$ \\
\hline $\begin{array}{c}\text { Technology } \\
(\mathrm{m})\end{array}$ & $\begin{array}{c}0.35 \\
\mu\end{array}$ & $0.18 \mu$ & $\begin{array}{c}0.18 \\
\mu\end{array}$ & $90 \mathrm{n}$ & $90 \mathrm{n}$ \\
\hline $\begin{array}{c}\text { Supply } \\
\text { Voltage (V) }\end{array}$ & 3.3 & \pm 0.75 & 1.5 & 0.8 & 0.8 \\
\hline $\begin{array}{c}\text { Power } \\
\text { Consumption } \\
(\mathrm{mW})\end{array}$ & 0.34 & - & $\begin{array}{c}0.32 \\
6\end{array}$ & 0.022 & 0.030 \\
\hline $\begin{array}{c}\text { Bandwidth } \\
(\mathrm{Hz})\end{array}$ & $\begin{array}{c}41.8 \\
\mathrm{M}\end{array}$ & $460 \mathrm{M}$ & $\begin{array}{c}340 \\
\mathrm{M}\end{array}$ & $320 \mathrm{M}$ & $1.2 \mathrm{G}$ \\
\hline $\begin{array}{c}\text { Nonlinearity } \\
\text { Error }(\%)\end{array}$ & 1.1 & 0.8 & 1.2 & 41 & 0.5 \\
\hline $\begin{array}{c}\text { Input range } \\
(\mu \mathrm{A})\end{array}$ & - & \pm 10 & \pm 40 & 100 & 100 \\
\hline
\end{tabular}

\section{Conclusion}

In this paper, a new low voltage low power BD-QFG RGC CM circuit is presented. It is the modified version of the conventional GD RGC CM. This proposed BD-QFG RGC presents a better linearity performance than the GD RGC. The proposed BD-QFG RGC has a $0.2 \%$ error current which $11.8 \%$ better than the GD RGC (12\%), a high output impedance (15 G $\Omega$ ) and a low power consumption (22.71 $\mu \mathrm{W})$. The proposed circuit enjoys a higher frequency value $(2.31 \mathrm{GHz})$ near to the frequency value of the GD-MOST $(2.36 \mathrm{GHz})$. The THD value of the proposed RGC CM is $0.4 \%$ which is $1.1 \%$ less than the conventional circuit (1.5\%). The mismatch variation of the proposed RGC is presented using Monte Carlo analysis and this layout simulation is also presented. An application of the proposed BD-QFG RGC $\mathrm{CM}$ is presented in the form of squarer circuit to ensure the workability of the proposed CM.

\section{References}

[1] M. B. et al, "Low voltage low power current comparator circuit," Int. Conf. Sc. Tech. Autom. Control Comput. Eng., pp. 168-172, 2018.

[2] R. G. et al, "Design of high speed and low power 4-bit comparator using FGMOS," AEU, vol. 76, pp. 125-131, 2017.

[3] R. Gupta and S. Sharma, "Quasi-floating gate MOSFET based low voltage current mirror," Microelectronics J., vol. 43, no. 7, pp. 439-443, 2012. 
[4] F. Kh. Et al, "Differential difference current conveyor using bulkdriven technique for ultra-low-voltage applications," Cir. Sys. Signal Process., vol. 33, no. 1, pp. 159-176, 2014.

[5] N. Raj et al, "Low power high output impedance high bandwidth QFGMOS current mirror," Microelectron. J., vol. 45, no. 8, pp. 1132-1142, 2014.

[6] F. Khateb, "Bulk-driven floating-gate and bulk-driven quasifloating-gate techniques for low-voltage low-power analog circuits design," $A E U$, vol. 68, no. 1, pp. 64-72, 2014.

[7] F. Khateb, "The experimental results of the bulk-driven quasifloating-gate MOS transistor," $A E U$, vol. 69 , no. 1, pp. 462-466, 2015.

[8] M. Bchir et al, "A Novel High Frequency Low Voltage Low Power Current Mode Analog to Digital Converter Pipeline," J. Low Power Electron., vol. 15, no. 4, pp. 368-378, 2019.

[9] B. Mounira et al, "Low voltage low power current mode analog to digital converter flash," in I. C. Sc. Elect. Tech. Info. Telec. SETIT, 2017, pp. 553-556.

[10] M. Bchir, I. Aloui, and N. Hassen, "A bulk-driven quasi-floating gate FVF current mirror for low voltage, low power applications," Integration, vol. 74, no. March, pp. 45-54, 2020.

[11] D. Yoon, J. E. Joo, and S. M. Park, "Mirrored Current-Conveyor Transimpedance Amplifier for Home Monitoring LiDAR Sensors," IEEE Sens. J., vol. 21, no. 5, pp. 5589-5597, 2021.

[12] Z. Qin, M. Shintani, K. Kuribara, Y. Ogasahara, and T. Sato, "Organic Current Mirror PUF for Improved Stability against Device Aging," IEEE Sens. J., vol. 20, no. 14, pp. 7569-7578, 2020.

[13] G. Maneesha et al, "A very high performance self-biased cascode current mirror for CMOS technology," A. Int. Circuits Signal Process., pp. 67-74, 2013.

[14] N. M. et al, "A Low Dropout Voltage Regulated Bulk-driven CMOS Current Mirror," in P.Computer Science, 2016, vol. 86, pp. 95-99.

[15] A. S. et al, "A compact class-AB bulk-driven quasi-floating gate current mirror for low voltage applications," Int. S. Commun. Inf. Technol., pp. 298-302, 2013.

[16] P. P. Mukherjee, "Design, Performance Analysis and Application of a Modified Improved MOS Regulated Cascode Circuit," Procedia Technol., vol. 4, pp. 477-481, 2012.

[17] G. Rockey et al, "Quasi-floating gate MOSFET based low voltage current mirror," Microelectron. J., vol. 43, no. 7, pp. 439-443, 2012.

[18] N. R. et al, "Low Voltage High Output Impedance Bulk-Driven Quasi-Floating Gate Self-Biased High-Swing Cascode Current Mirror," C. S. Signal Process., vol. 35, no. 8, pp. 2683-2703, 2016.

[19] M. Doreyatim et al, "A low-voltage gain boosting-based current mirror with high input / output dynamic range," Microelectronics J., vol. 90, pp. 88-95, 2019.

[20] L. Safari et al, "A Low-Voltage Low-Power Resistor-Based Current Mirror and Its Applications," J. Circuits, Syst. Comput., vol. 26, no. 11, p. $1750180,2017$.

[21] I. Aloui, N. Hassen, and K. Besbes, "A CMOS current mode four quadrant analog multiplier free from mobility reduction," $A E U$ Int. J. Electron. Commun., vol. 82, pp. 119-126, 2017.

[22] A. Naderi and A. Khoei, "High Speed, Low Power FourQuadrant CMOS Current-Mode Multiplier," IEEE Int. Conf. Electron., no. 2, pp. 1308-1311, 2007.

[23] M. A. AL-Absi and I. A. As-Sabban, "A CMOS current-mode squaring circuit free of error resulting from carrier mobility reduction," Analog Integr. Circuits Signal Process., vol. 81, no. 1, pp. 23-28, 2014. 